\title{
Caveolin-1 facilitated KCNA5 expression, promoting breast cancer viability
}

\author{
CHAO QU ${ }^{1-3 *}$, JIA SUN $^{1 *}$, YING LIU $^{1,4^{*}}$, XIAOBO WANG ${ }^{3}$, LIFEN WANG $^{5}$, CHAO HAN $^{1,2}$, QIAN CHEN $^{1}$, \\ TIANHUI GUAN ${ }^{1}$, HONGYAN LI ${ }^{1}$, YEJUN ZHANG ${ }^{1}$, YANG WANG ${ }^{1,6}$, JIA LIU ${ }^{1}$, WEI ZOU ${ }^{1}$ and JING LIU $^{2}$ \\ ${ }^{1}$ College of Life Science, Liaoning Normal University, Dalian, Liaoning $116029 ;{ }^{2}$ Centre for Regenerative Medicine, \\ First Affiliated Hospital of Dalian Medical University, Dalian, Liaoning 116085; ${ }^{3}$ No. 210 Hospital of Chinese People's \\ Liberation Army, Dalian, Liaoning 116021; ${ }^{4}$ Institute for Translational Medicine, Qingdao University, Qingdao, \\ Shandong 266021; ${ }^{5}$ The Second Affiliated Hospital of Dalian Medical University, Dalian, Liaoning 116027, P.R. China; \\ ${ }^{6}$ Department of Medical Microbiology and Immunology, Creighton University School of Medicine, Omaha, NE 68178, USA
}

Received October 19, 2017; Accepted April 16, 2018

DOI: $10.3892 /$ ol.2018.9261

\begin{abstract}
Potassium voltage-gated channel subfamily A member 5 (KCNA5) is a voltage-gated potassium channel protein encoded by the KCNA5 gene. A large number of studies have shown that KCNA5 is associated with the survival of malignant tumors, including breast cancer, but the detailed mechanism remains inconclusive. Our previous study found that KCNA5 is co-expressed with a scaffolding protein, caveolin-1 in MCF-10A-neoT non-tumorigenic epithelial cell. In the present study, KCNA5 and caveolin-1 were expressed in breast cancer tissues and cell lines. Exposing MCF-10A-neoT to $2 \mathrm{mM}$ of methyl- $\beta$-cyclodextrin, an agent to disrupt caveolae and lipid rafts led to a downregulation of caveolin-1 that reduced the expression of KCNA5. Furthermore, following caveolin-1 knockdown, the expression of KCNA5 was decreased in MDA-MB-231 human breast cancer and MCF-10A-neoT non-tumorigenic epithelial cell
\end{abstract}

Correspondence to: Professor Wei Zou, College of Life Science, Liaoning Normal University, 3 Teaching Building, 1 Liushunanjie, Dalian, Liaoning 116029, P.R. China

E-mail:weizou60@hotmail.com

Professor Jing Liu, Centre for Regenerative Medicine, First Affiliated Hospital of Dalian Medical University, 193 Lianhelu Xigang, Dalian, Liaoning 116085, P.R. China

E-mail: liujing.dlrmc@hotmail.com

*Contributed equally

Abbreviations: $\mathrm{K}_{\mathrm{v}}$, voltage-gated $\mathrm{K}^{+}$channel; MCF-10A-neoT, non-tumorigenic epithelial cell line; MCF7 and MDA-MB-231, human breast cancer cell lines; MAPK, ras/mitogen-activated protein kinase; AKT, serine/threonine protein kinase B; Cav-1, caveolin-1; PCNA, proliferation cell nuclear antigen; $\mathrm{M} \beta \mathrm{CD}$, methyl- $\beta$-cyclodextrin

Key words: caveolin-1, KCNA5, human breast cancer cells, $\mathrm{PI} 3 \mathrm{~K} / \mathrm{AKT}$ pathway lines. In subsequent experiments, the MTT assay showed that increased caveolin-1 and KCNA5 expression promoted the survival of MCF-7 human breast cancer cells, but cell survival was not affected following KCNA5 overexpression alone. Using small interfering RNA technology, KCNA5-silenced MCF-10A-neoT cells were established and a decreased level of phosphorylated-AKT serine/threonine kinase (AKT) was observed in the cells compared with the parental cells. Overall, these results suggested that caveolin-1 facilitated KCNA5 expression and may be associated with AKT activation.

\section{Introduction}

Voltage-gated potassium $\left(\mathrm{K}_{\mathrm{v}}\right)$ channels play a key role in several physiological processes, such as the membrane potential maintainance, $\mathrm{Ca}^{2+}$ signaling, and the cell volume regulation. In addition, they are also involved in cell survival and migration (1). Most studies have focused on the function of $\mathrm{K}_{\mathrm{v}}$ channels in viability of tumor cells, particularly tumor cells of epithelial origin, such as human mammary epithelial cell $(2,3)$. Accumulated evidence indicated that the expression of $\mathrm{K}_{\mathrm{v}}$ channels is related to tumor development (4). However, the mechanism of $\mathrm{K}_{\mathrm{v}}$ channels in these cells is still unknown. Thus, many drugs and toxins that specifically block $\mathrm{K}_{\mathrm{v}}$ channels have been tested for their effect on cell proliferation. Among the $\mathrm{K}_{\mathrm{v}}$ channels subunits, potassium voltage-gated channel subfamily A member 5 (KCNA5) specifically has been shown to be involved in the viability and apoptosis of oligodendrocytes, hippocampus microglia, macrophages and human mammary epithelial cells (5-7). It has reported that most tumor cells had increased expression of KCNA5 (8). Furthermore, the transition of quiescent cells into the $\mathrm{G}_{1}$ phase is accompanied by the over-expression of Kv1.3 and KCNA5 proteins in rat oligodendrocyte precursor cells. Blocking KCNA5 sufficiently slowed the viability of astrocytes rather than oligodendrocytes, indicating that this channel may play different roles in different cells.

AKT phosphorylation is transduced and amplified through downstream kinase cascades, inducing cell survival, growth, differentiation as well as metabolic changes (2). In 
general, $\mathrm{K}_{\mathrm{v}}$ channels are modulated by mitogenic signals, such as growth factor-mediated signaling $(8,9)$. In HEK293 cells, IGF-1 induces the expression of several $\mathrm{K}_{\mathrm{v}}$ channels in response to mitogenic signals (10). The mitogenic stimulation of $\mathrm{G}_{0}$ phase activates $\mathrm{K}^{+}$channels, drives the cells into $\mathrm{G}_{1}$ phase, and then initiates proliferation $(3,11) . \mathrm{K}_{\mathrm{v}}$ channels play a major role in advancing the cell cycle when activated by mitogenic factors $(12,13)$. Interestingly, $\mathrm{K}_{\mathrm{v}}$ channels are upstream modulators of growth factor-mediated MAPK and PI3K/Akt pathways $(14,15)$. And many current studies suggest that $\mathrm{K}^{+}$channels involved in the initial mitogenic signaling events occur at the membrane level. Moreover, the activation of $\mathrm{K}^{+}$channels may lead to receptor clustering, thereby facilitating transmembrane signaling (2).

Lipid rafts support numerous cellular events in membrane trafficking and signal transduction mediated by multiple membrane proteins (16-18). Caveolae are a type of lipid raft containing specific scaffolding proteins, like caveolin (19). Various lipid rafts share similar lipid proteins (20-22). The caveolin family has three members, including caveolin-1 (Cav-1), Cav-2 and Cav-3, of which Cav-3 is restricted to muscle cells $(23,24)$. Many signaling molecules are directly related to Cav-1 $(25,26)$. Some proteins have been well-characterized, including $\mathrm{Kv}$ channels that interact with caveolin, which are to concentrate the cargo proteins in the caveolae $(27,28)$. It has been reported that the expression and localization of Kv channels are important to play their function fully in cells (29). Numerous Kv channels localize to the lipid raft domains and/or caveolae in the plasma membrane. KCNA5 has been found in raft microdomains and their functions are influenced by lipid-protein interactions. Martens et al showed that KCNA5 can localize to caveolae microdomains, and KCNA5 was associated with caveolae (27). However, we are unknown for the mechanisms controlling their interactions and the physiological functions of this localization. Recent research has found a role of Cav-1 in transporting proteins to the cell membrane (30). And according to some recent studies, Cav-1 regulates proteins that co-localize with it, such as estrogen receptor (ER), KCNA5, and desmoglein 2 (Dsg2) (31-33). However, the role of Cav-1 in mediating the membrane localization of KCNA5 channel has not been elucidated.

Our previous study demonstrated that $\mathrm{K}_{\mathrm{v}}$ channels were required for the viability of the normal MCF-10A-neoT cells (7). In this study, we described that KCNA5 and Cav-1 co-localize in the cytoplasm of MCF-7 human breast cancer cells. The study also found that the knockdown KCNA5 inhibited the PI3K/AKT signaling pathway in MCF-10A-neoT cells, and cells upregulated with Cav-1 and KCNA5 promoted survival in MCF-7 cells through PI3K/AKT signaling. In addition, it was showed that the downregulation of Cav-1 decreased the expression of KCNA5, indicating that Cav-1 was involved in the KCNA5-promoted survival of human mammary cells.

\section{Materials and methods}

Plasmids and antibodies. The KCNA5 plasmid was from Dr Jie Zheng (University of California, Davis). The Cav-1 plasmid and siRNA plasmid specific for Cav-1 (target sequence Oligo 1, 5'-ACCTCATTAAGAGCTTCCTGATTGAGTCAA GAGCTCAATCAGGAAGCTCTTAATTT-3', Oligo 2 ,
5'-CAAAAAATTAAGAGCTTCCTGATTGAGCTCTTGACT CAATCAGGAAGCTCTTAATG-3') were obtained from the Cancer Center at Creighton University.

Anti-KCNA5 (rabbit polyclonal, 1:500; EMD Millipore, Billerica, MA, USA), anti-Cav-1 (mouse monoclonal, 1:1,000, Santa cruz biotechnology), anti-p-MAPK (mouse monoclonal, 1:1,000), anti-MAPK (rabbit polyclonal, 1:1,000), anti-p-AKT (rabbit monoclonal, 1:1,000) (all from Cell Signaling Technology, Danvers, MA, USA), anti-AKT (goat polyclonal, 1:1,000; Santa Cruz Biotechnology, Inc., Santa Cruz, CA, USA), 1:500), anti-PCNA (mouse monoclonal, 1:500) and anti- $\beta$-actin (mouse monoclonal, 1:1,000) (all from Wuhan Boster Biological Technology, Ltd., Wuhan, China). HRP-conjugated goat anti-rabbit, anti-mouse or anti-goat specific secondary antibody (1:6,000; Zhongshan Golden Bridge Biotechnology, Beijing, China).

Patients. A total of 23 breast cancer tissues were obtained from patients in the First Affiliated Hospital of Dalian Medical University. All the patients were females aged 29-83 with infiltrative non-specific breast cancer. The selected tissue samples express both ER $\alpha$ and ER- $\alpha 36$ under immunofluorescence observation, and without any radiation, chemotherapy, or endocrinotherapy treatment before surgical resection. We got the patients' relatives written informed consent for the procedures, which were also approved by the Ethics Committee on the Use of Human Subjects (the First Affiliated Hospital of Dalian Medical University).

Cell culture and transfection. The MCF-10A-neoT, MCF-7 and MDA-MB-231 cells were purchased from ATCC (Rockville, MD, USA). Stable clones (designated as MCF-10A-neoT ${ }^{\mathrm{CE}}$ ) were established as described in our previous study $(34,35)$. The MCF-10A-neoT and MCF-10A-neoT ${ }^{\mathrm{CE}}$ cells were cultured in DMEM/F12 medium (Gibco; Thermo Fisher Scientific, Inc., Waltham, MA, USA) supplemented with $5 \%$ horse serum (HyClone, Logan, UT, USA), penicillin (100 U/ml), streptomycin $(100 \mu \mathrm{g} / \mathrm{ml})$ (both from SigmaAldrich; Merck KGaA, Darmstadt, Germany), hydrocortisone $\left(1.4 \times 10^{-6} \mathrm{M}\right.$; HyClone), insulin $(10 \mu \mathrm{g} / \mathrm{ml})$, cholera toxin $(100 \mathrm{ng} / \mathrm{ml})$ and EGF $(20 \mathrm{ng} / \mathrm{ml})$ (both from Sigma-Aldrich, Merck KGaA). MCF7, MDA-MB-231 were cultured in RPMI1640 supplemented with $10 \%$ fetal bovine serum (both from Gibco; Thermo Fisher Scientific, Inc.), penicillin (100 U/ml), and streptomycin $(100 \mu \mathrm{g} / \mathrm{ml})$. All cells were maintained in a humidified atmosphere at $37^{\circ} \mathrm{C}$ in $5 \% \mathrm{CO}_{2}$. Lipofectamine $2000^{\mathrm{TM}}$ (Invitrogen; Thermo Fisher Scientific, Inc.) was used for cell transfection according to the manufacturer's instructions. After 24-48 h of transfection and subsequent culture in $1 \mu \mathrm{M}$ wortmannin or $50 \mu \mathrm{M}$ Ly294002 (Sigma-Aldrich, Tokyo, Japan), cells were harvested for western blot analysis or 3-(4,5-dimethylthiazol-2-yl)-2,5-diphenyltetrazolium bromide (MTT) assay. Methyl- $\beta$-cyclodextrin $(\mathrm{M} \beta \mathrm{CD})$ was used to disrupt caveolae. MCF-10A-neoT cells pretreated with M $\beta C D$ (2 mM) for $90 \mathrm{~min}$ were used for immunofluorescent microscopy analysis.

Western blotting. Cells were harvested and then lysed in a cold lysis buffer $\left(20 \mathrm{mmol} \mathrm{L}^{-1}\right.$ Tris- $\mathrm{HCl}, \mathrm{pH} 7.5,70 \mathrm{mmol} \mathrm{L}^{-1} \mathrm{NaCl}$, $0.1 \%$ SDS, $1 \%$ sodium deoxycholate, $1 \%$ Triton $\mathrm{X}-100$ and 
1\% PMSF) to extract protein (35). The concentration of total protein was determined by the Bradford method. The protein samples were then subjected to $10 \%$ SDS-PAGE. After electrophoresis, protein bands were transferred to a polyvinylidene fluoride (PVDF) membrane, and then blocked in PBS-T ( $\mathrm{pH} 7.4$ ) containing 5\% dried skim milk. Then the PVDF membrane was probed with the specified primary antibody, followed by the appropriate secondary antibody, and finally visualized using the ECL ${ }^{\mathrm{TM}}$ which is a Western blotting chemiluminescent reagent kit (Amersham Biosciences, Piscataway, NJ, USA) according to the manufacturer's instructions. Immunoblot data were quantified using ImageJ software (NIH, Bethesda, MD, USA). The region of interest was marked and measured in every lane, and the background was subtracted to give the final band intensity.

RNA interference. The small interfering RNAs (siRNA) against the KCNA5 mRNA sequence (GenBank accession number NM_002234) were predesigned and synthesized in Takara (Dalian, China), meanwhile, an unrelated siRNA serving as a negative control was randomly designed. The sequences of the KCNA5 siRNA vector plasmid (target sequence Oligo 1, 5'-GATCCACCAGGGAACCCATTTCTCTCTGTGAAGCCA CAGATGGGAGAGAAATGGGTTCCCTGGTTTTTTTAT-3', target sequence Oligo 2, 5'-CGATAAAAAAACCAGGGA ACCCATTTCTCTCCCATCTGTGGCTTCACAGAGAGAA ATGGGTTCCCTGGTG-3'), and the negative control siRNA plasmid (target sequence Oligo 1, 5'-GATCCAGATCCTCAC GATACCGTCTCTGTGAAGCCACAGATGGGAGACGGTA TCGTGAGGATCTTTTTTTAT-3', target sequence Oligo 2 , 5'-CGATAAAAAAAGATCCTCACGATACCGTCTCCCATC TGTGGCTTCACAGAGACGGTATCGTGAGGATCTG-3').

Knocking down the basal expression levels of KCNA5 or Cav-1 in MCF-10A-neoT or MDA-MB-231 cells were performed using the respective siRNAs, as well as scrambled control siRNA. The cells were transfected with Lipofectamine $^{\text {TM }} 2000$ (Invitrogen; Thermo Fisher Scientific, Inc.) and $4 \mu \mathrm{g}$ of Cav-1-siRNA according to the manufacturer's recommendation. After $48 \mathrm{~h}$ of transfection, culture medium with G418 (Amresco, Solon, OH, USA) was added to the cells. The cells were harvested when the monoclone formed and were processed for further analysis.

Survival assay. For the MTT assay, $20 \mu 1$ MTT reagent was added to each well and incubated for $4 \mathrm{~h}$. The supernatant was discarded and replaced with DMSO to dissolve the formazan product, which was measured by a spectrophotometric plate reader at $490 \mathrm{~nm}$.

Hematoxylin and eosin staining. Human breast cancer specimens were fixed for 1 week in $4 \%(\mathrm{w} / \mathrm{v})$ PBS-buffered formaldehyde solution at room temperature, dehydrated using graded ethanol, and embedded in Paraplast (Sherwood Medical, Mahwah, NJ, USA). Sections were stained with hematoxylin and eosin, then deparaffinized with xylene. All sections were studied by using a AxioVision zeiss (Olympus, Tokyo, Japan) microscope.

Immunofluorescent staining. Cells were grown on glass coverslips coated with poly-L-lysine, and cultured for $24 \mathrm{~h}$.
The cells were then fixed in $4 \%$ ice cold paraformaldehyde for 15-20 min. Cells were permeabilized with PBS containing $0.1 \%$ Triton $\mathrm{X}-100$ for $10 \mathrm{~min}$. After blocking with $5 \%$ bovine serum albumin (BSA) for $1 \mathrm{~h}$, the cells were then incubated with appropriate antibodies at $4^{\circ} \mathrm{C}$ overnight, followed by further incubation with fluorescein-conjugated affinipure goat anti-mouse antibodies (1:300) and Rhodamine-conjugated affinipure goat anti-rabbit antibodies (1:300) (both from Zhongshan Golden Bridge Biotechnology). Nucleus was stained using DAPI (0.2 $\mu \mathrm{g} / \mathrm{ml}$; Sigma-Aldrich, Merck KGaA). Fluorescence was imaged with a Bio-Rad MRC 600 confocal imaging system. Images were taken with a Leica TCS SP2 multiphoton confocal microscope.

Statistical analysis. All data were expressed as the mean $\pm \mathrm{SE}$ Unpaired Student's t-test was used to test for statistical significance between the control and test groups. Comparisons of multiple groups were analyzed using a one- or two-way ANOVA followed by post hoc Tukey's test. P-value $<0.05$ was considered significance.

\section{Results}

Cav-1 and KCNA5 are co-expression in human breast cancer cell lines. Cav-1 and KCNA5 have been shown to be related to epithelial cell growth and survival, and are abnormally expressed in malignant tumors including breast cancer. The study demonstrated that $\mathrm{K}_{\mathrm{v}}$ channels activate MAPK and AKT pathways, and Cav-1 and KCNA5 were co-localized in the membrane of MCF-10A-neoT non-tumorigenic epithelial cell line (36). The mechanism by which KCNA5 activates AKT signaling has not been fully determined, but it has been speculated to be activated through Cav-1. To examine the expression of KCNA5 and Cav-1 in breast cancer tissues and cells, hematoxylin and eosin staining was used to determine the region of cancer (Fig. 1A). Next, the study tried to determine whether there was co-expression between Cav-1 and KCNA5 in breast cancer tissues and cells. Cav-1 and KCNA5 both expressed and co-expressed in breast cancer tissues (Fig. 1A) and cells (Fig. 1B). After enlarging MCF-7 fluorescence images, it could be found KCNA5 and Cav-1 are co-expression in cytoplasm and cell membrane, but Cav-1 mainly expressed in cytoplasm of MCF-7 (Fig. 1B). Here, we examined KCNA5 and Cav-1 expression in MCF-10A-neoT, MCF-7 and MDA-MB-231 cells. Western blot analysis showed that both KCNA5 and Cav-1 expression are lower in MCF-7 breast cancer cell compared with MCF-10A-neoT non-tumorigenic epithelial cell and KCNA5 and Cav-1 expressions are significantly higher in MDA-MB-231 breast cancer cell than MCF-7 (Fig. 1C).

KCNA5 and Cav-1 are coupled in caveolae of human breast cancer cells. Our lab already has generated stable MCF-10A-neoT ${ }^{\mathrm{CE}}$, which is a Cav-1 knockdown cell line (34). Compared with control cells, immunofluorescence (Fig. 2) and immunoblot (Fig. 3A) both showed reduced expression of KCNA5 in MCF-10A-neoT ${ }^{\mathrm{CE}}$ cells. Quantification of the immunoblots demonstrated that the siRNA reduced Cav-1 by $\sim 80 \%$ and KCNA5 by $\sim 50 \%$ in MCF-10A-neoT ${ }^{\mathrm{CE}}$. To further confirm the association of Cav-1 with KCNA5, 


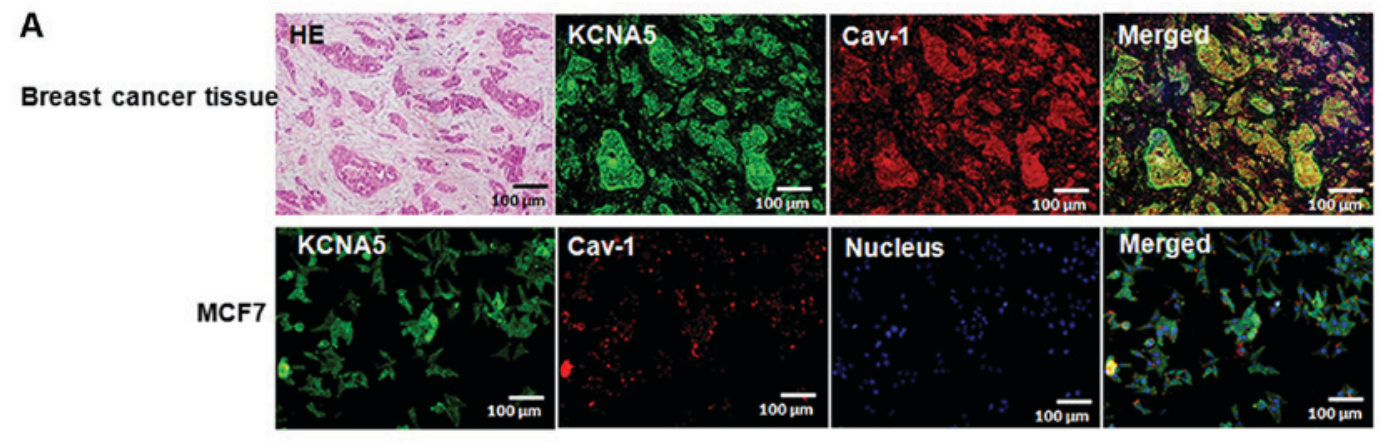

B

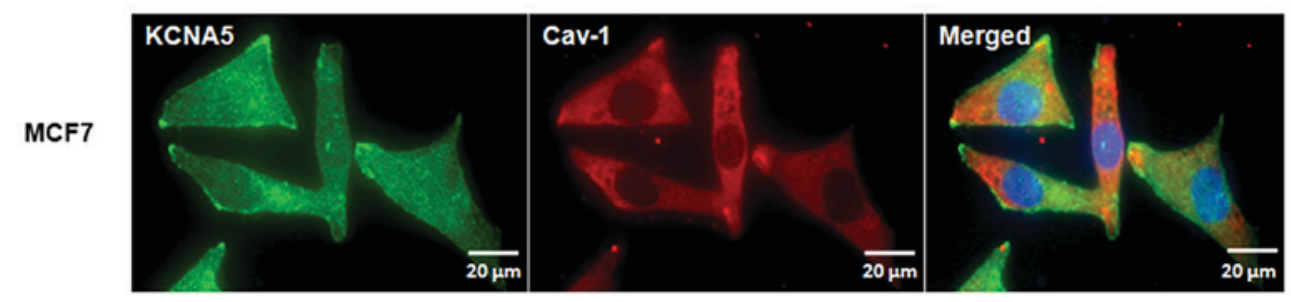

C
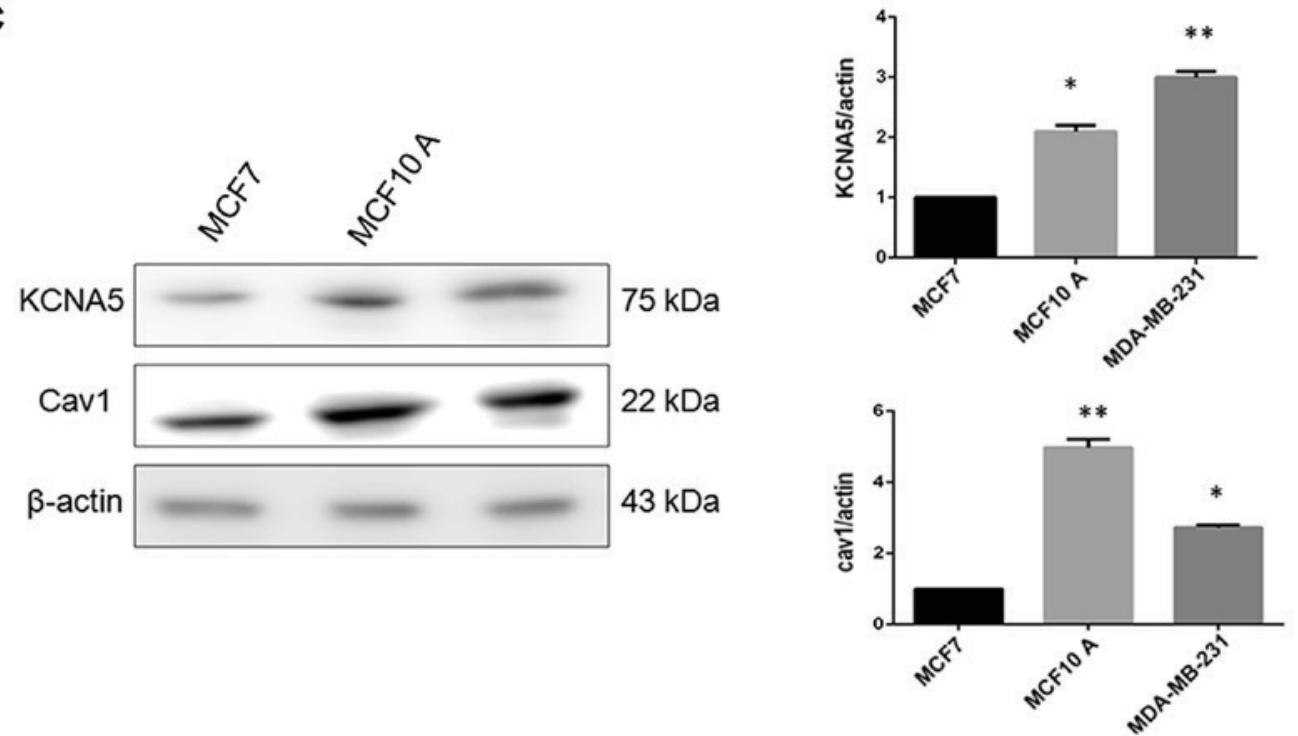

Figure 1. The co-expression of KCNA5 and Cav-1 in human breast cancer cells. Hematoxylin and eosin staining for human breast cancer tissue. (A) Immunofluorescence staining with KCNA5 and Cav-1 antibody in breast cancer tissue (KCNA5 for green, Cav-1 for red, Hoechst 33324 to label nuclear DNA). Bar, $100 \mu \mathrm{m}$. (B) MCF-7 human breast cancer cells (KCNA5 for green, Cav-1 for red, Hoechst 33324 to label nuclear DNA), bar, $100 \mu \mathrm{m}$ or bar, $20 \mu \mathrm{m}$. Representative images were obtained by a Leica TCS SP 2 multiphoton confocal microscope. (C) Western blot analyses of Cav-1 and KCNA5 expression in MCF-7, MDA-MB-231 and MCF-10A-neoT cells. The experiment was repeated four times; SE. " $\mathrm{P}<0.05$ and ${ }^{* *} \mathrm{P}<0.01$ for MCF-10A-neoT and MDA-MB-231 cells vs. MCF-7 cells. KCNA5, potassium voltage-gated channel subfamily A member 5; Cav-1, caveolin-1.

MCF-10A-neoT was treated with $2 \mathrm{mM} \mathrm{M} \beta \mathrm{CD}$ to disrupt caveolae and lipid rafts, and it showed that the expressions of Cav-1 and KCNA5 both significantly decreased in the cell membrane of MCF-10A-neoT (Fig. 2). Collectively, our data demonstrated that the knockdown of Cav-1 reduced KCNA5 levels in MCF-10A-neoT. Similar results were also obtained in MDA-MB-231 (Fig. 3B), which indicated that Cav-1 increased KCNA5 expression in human breast cancer cells. However, the expression of Cav-1 in MCF-10A-neoT-siKCNA5 and MCF-10A-neoT-vector were not changed (Fig. 3C). We over-expressed KCNA5 in MCF7 cell that Cav-1 is low expression, however, we found that overexpression of KCNA5 does not affect Cav-1 expression in MCF7 cells (Fig. 3D).

KCNA5 promotes human breast cancer cells survival through Cav-1. The PI3K/AKT and MAPK signaling pathways are the two major pathways involved in the regulation of cell viability (37). To test the function of Cav-1 and KCNA5 in MCF-7 cells, we made a Cav-1 and KCNA5 vectors co-transfection. Intriguingly, cells with increased Cav-1 and KCNA5 promoted cell survival, and the AKT signaling pathway inhibitor, Wortmanin significantly inhibited cell survival in MCF-7 (Fig. 4A-C). What's more, cells with both upregulated Cav-1 and KCNA5 had increased AKT activation (Fig. 4D). Therefore, we believe KCNA5 involves in cells survival, only when Cav-1 is overexpression. And PI3K/AKT signaling pathway involves in KCNA5-dependent cell survival.

To determine whether KCNA5 survival through Cav-1 in human breast cancer cell lines, we used MCF-10A-neoT as a cell model, which has an overexpression Cav-1 showing in Fig. 1C. Besides, by knocking down KCNA5 in MCF-10A-neoT, we detected whether or not the reduced expression of KCNA5 


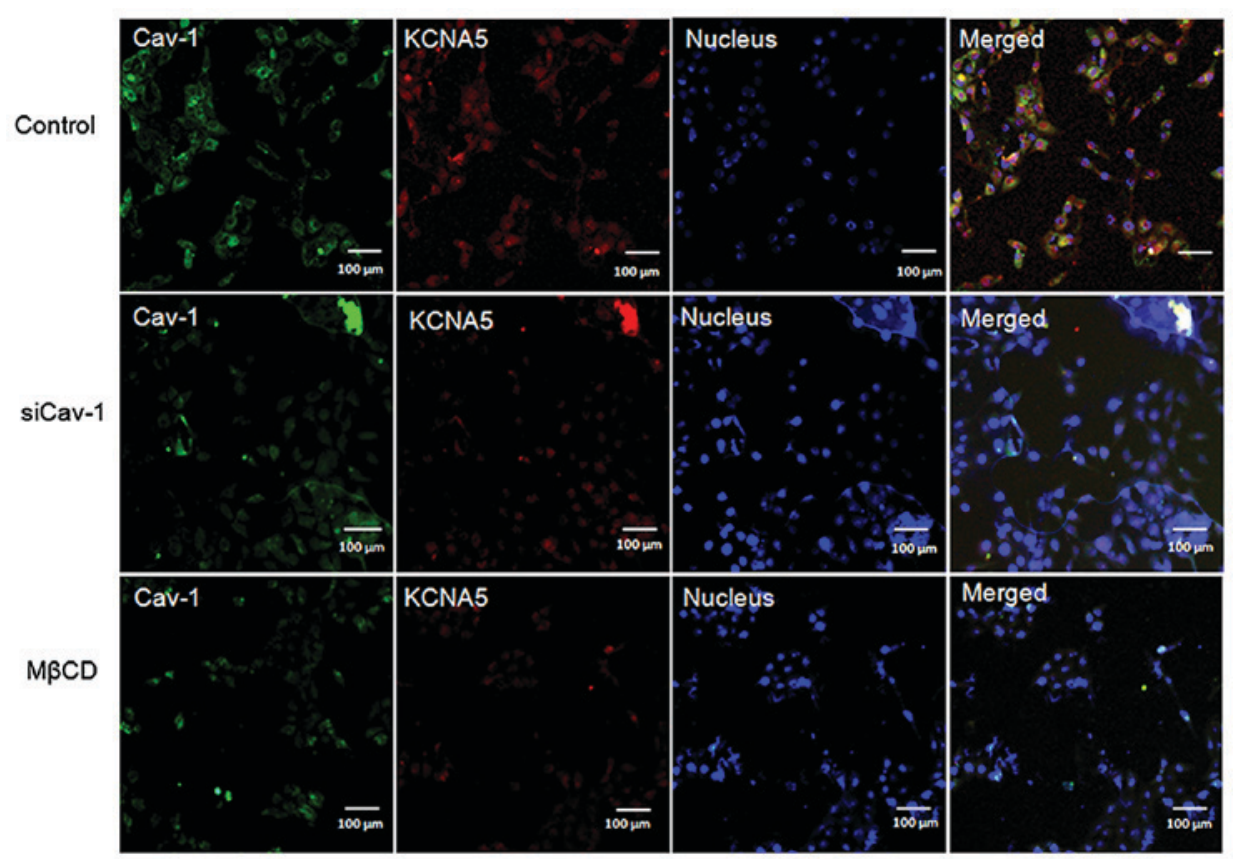

Figure 2. KCNA5 and Cav-1 are coupled together in caveolae. Immunofluorescence staining with KCNA5 and Cav-1 antibody in MCF-10A-neoT. Control: Co-expression of KCNA5 (red) and Cav-1 (green) in MCF-10A-neoT cells; Cav (-/-): Co-expression of KCNA5 (red) and Cav-1 (green) in Cav-1 expression knocked-down cells, MCF-10A-neoT ${ }^{\mathrm{CE}}$; M $\beta C D: 2 \mathrm{mM}$ methyl- $\beta$-cyclodextrin destroy the caveolae structure, and examined co-expression of KCNA5 (red) and Cav-1 (green) in MCF-10A-neoT, Bar, $100 \mu \mathrm{m}$. KCNA5, potassium voltage-gated channel subfamily A member 5; Cav-1, caveolin-1.

A
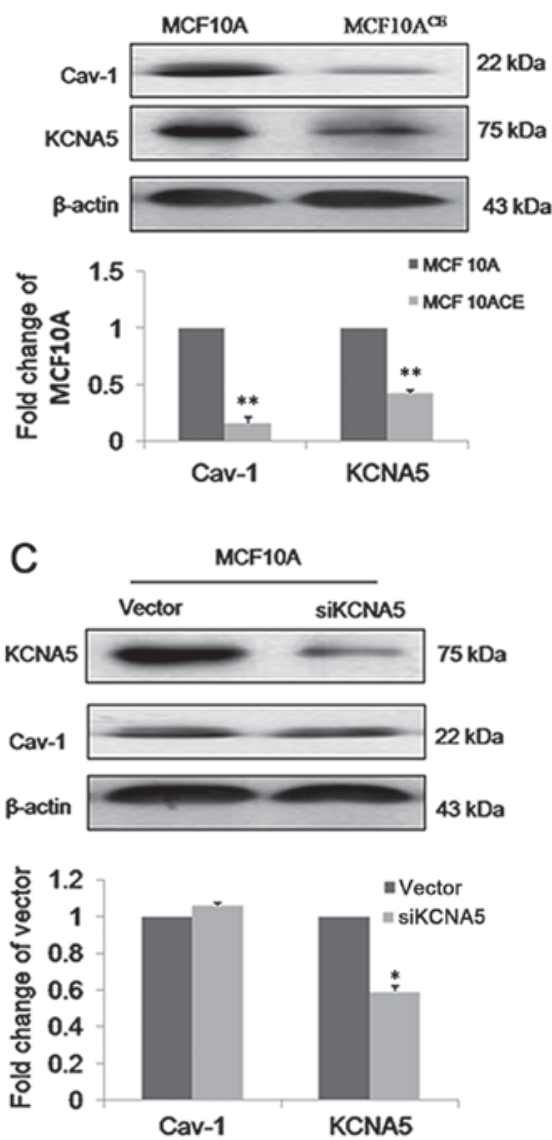

B
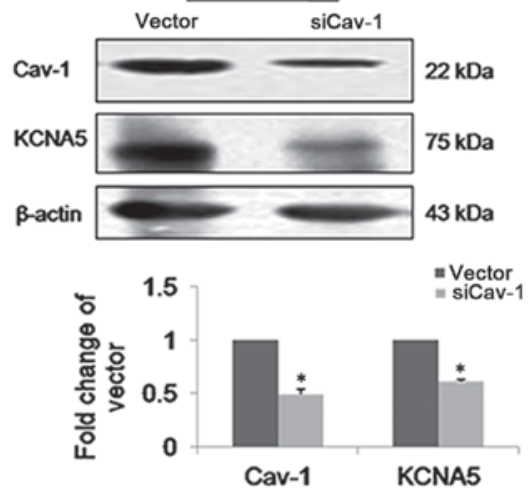

D
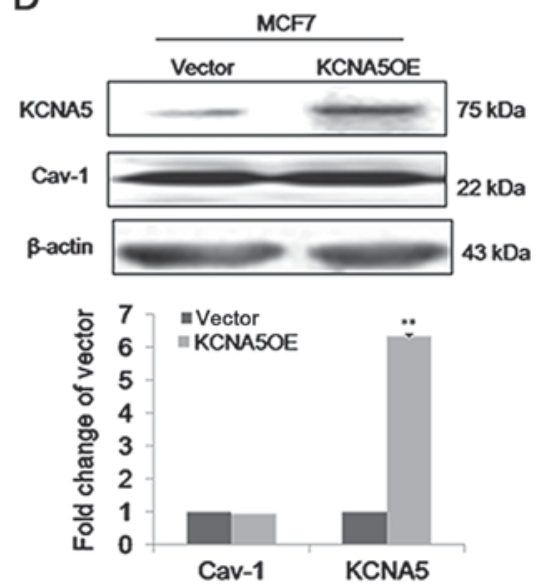

Figure 3. Effect of Cav-1 on the expression of KCNA5. (A) Western blotting analyzed the expression of KCNA5 in MCF-10A-neoT and Cav-1 expression knocked-down cells, MCF-10A-neoT ${ }^{\mathrm{CE}}$. (B) Cav-1 high expression MDA-MB-231 cells were transfected with the Cav-1 siRNA plasmid, western blot analyses of KCNA5 expression. (C) Western blot analyses of Cav-1 expression in MCF-10A-neoT cells and KCNA5 expression knocked-down cells MCF-10A-neoT-siKCNA5. (D) KCNA5 low expression MCF-7 cells were transfected with KCNA5 expression vector for $48 \mathrm{~h}$, western blot analyses of Cav-1 expression. The experiment was repeated three times. " $\mathrm{P}<0.05$ and ${ }^{* *} \mathrm{P}<0.01$ vs. vector. KCNA5, potassium voltage-gated channel subfamily A member 5; Cav-1, caveolin-1. 

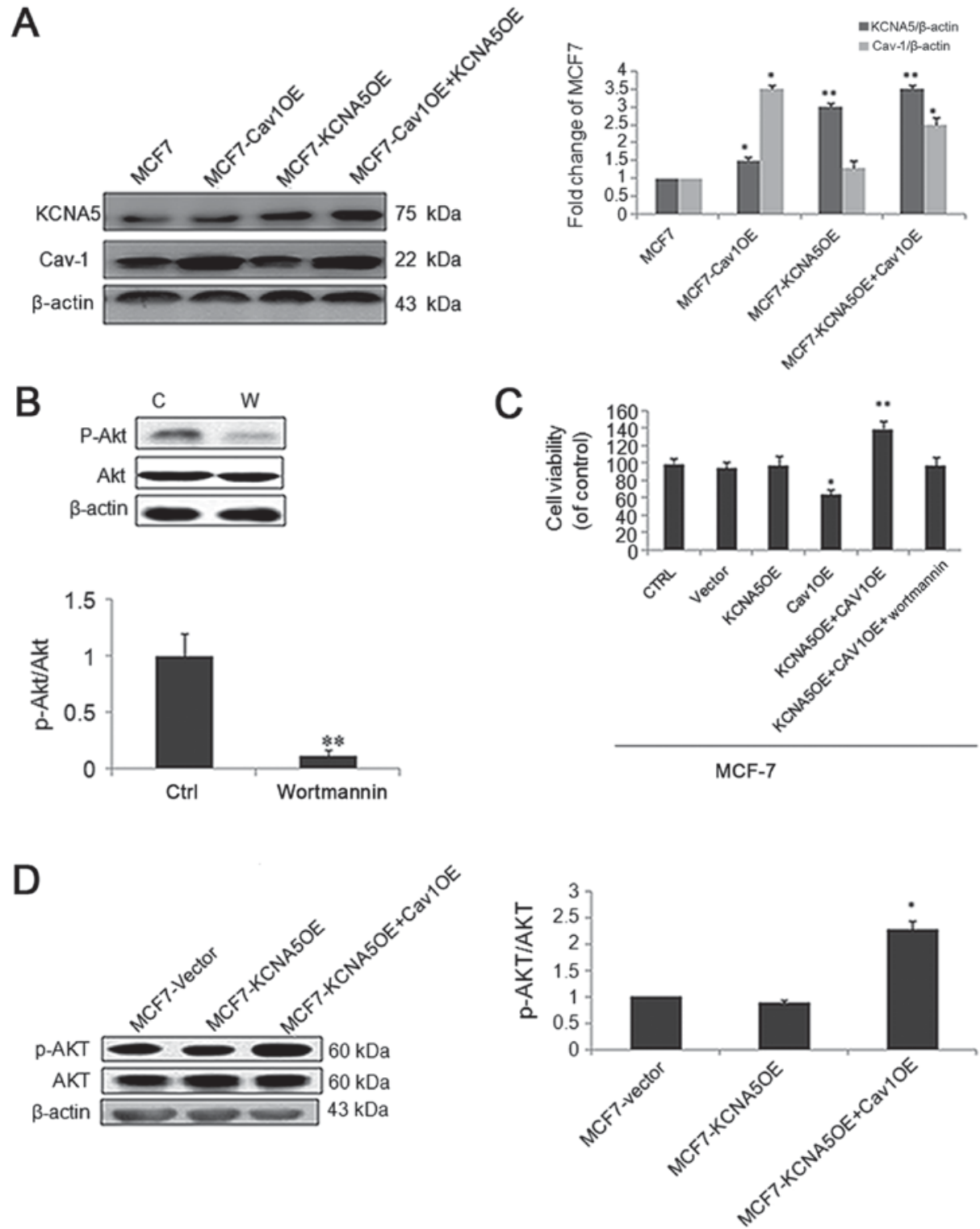

Figure 4. KCNA5 promoted human breast cancer cells survival is dependent on Cav-1. MCF-7 cells were transfected with KCNA5 or Cav-1 vector alone, or co-transfected with both Cav-1 and KCNA5 vectors for 48 h. (A) Western blot analyses of KCNA5 and Cav-1 expression. (B) Western blot analysis of Akt phosphorylation after wortmannin treatment. (C) Cells upregulated Cav-1 and KCNA5 were treated with wortmannin $(1 \mu \mathrm{M})$ for $24 \mathrm{~h}$. The survival of MCF-7 cells was examined by the MTT assay. (D) Western blot analysis of AKT phosphorylation in different MCF-7 variants; control cells MCF-7-vector (transfected with the empty expression vector); MCF-7-KCNA5 OE (transfected with the KCNA5 expression vector); MCF-7-KCNA5 OE+Cav-1 OE (co-transfected with the KCNA5 and Cav-1 expression vector. The columns represent the means of five experiment; $\mathrm{SE}$. ${ }^{*} \mathrm{P}<0.05$ and ${ }^{* *} \mathrm{P}<0.01$ for cells transfected with Cav-1 or KCNA5 expression vectors vs. empty expression vector. KCNA5, potassium voltage-gated channel subfamily A member 5; Cav-1, caveolin-1.

inhibited cell survival through the PI3K/AKT and/or MAPK signaling pathways in MCF-10A-neoT. As shown in Fig. 5A, there was no obvious change of p-MAPK in the two cell lines, but the level of p-AKT was decreased.

To study whether KCNA5 directly promote the cells survival through PI3K/AKT pathway, we observed the effects of wortmannin, LY 294002, inhibitors of AKT and PI3K on expression of KCNA5 and phospho-AKT protein in both MCF-10A-neoT and MCF-10A-neoT-siKCNA5 cell lines. As shown in Fig. 5B, the level of KCNA5 was not changed after treatment with two inhibitors in the MCF-10A-neoT. MCF-10A-neoT treated with both inhibitors, AKT activation was inhibited compared with control (Fig. 5C). In addition, compared with control, the level of p-AKT was significantly inhibited by wortmannin, however, LY29002 had no effect on p-AKT in the MCF-10A-neoT-siKCNA5 cells (Fig. 5D). Finally, we summarized the possible mechanism of how KCNA5 enhances breast cancer cells viability in Fig. 6.

\section{Discussion}

The study demonstrated that KCNA5 and Cav-1 co-expression in breast cancer and normal mammary tissue, and in MCF-10A-neoT and MCF-7 cells. And it was expounded that KCNA5 and Cav-1 upregulation in human breast cancer cells promoted cell survival and activated AKT. Furthermore, KCNA5 knockdown with siRNA resulted in a decreased phosphorylation of AKT, but not inhibit MAPK signaling in MCF-10A-neoT. Additionally, Cav-1 knockdown led to decreased KCNA5 expression in MCF-10A-neoT and 
A
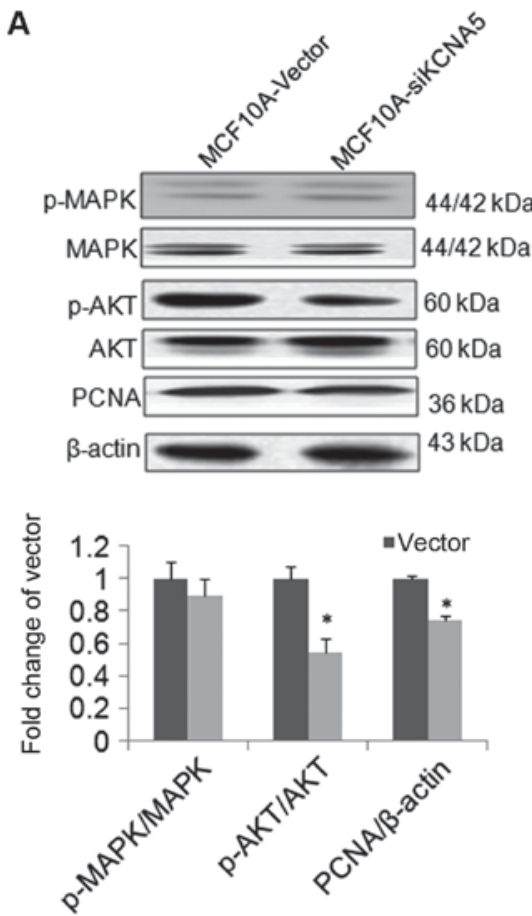

C
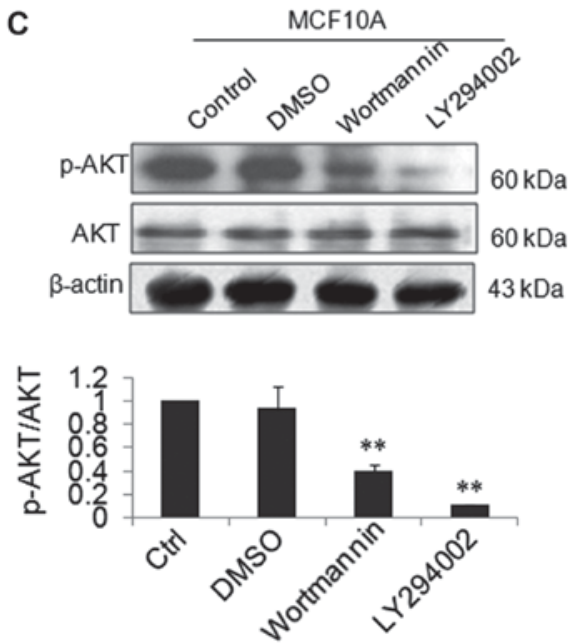

B
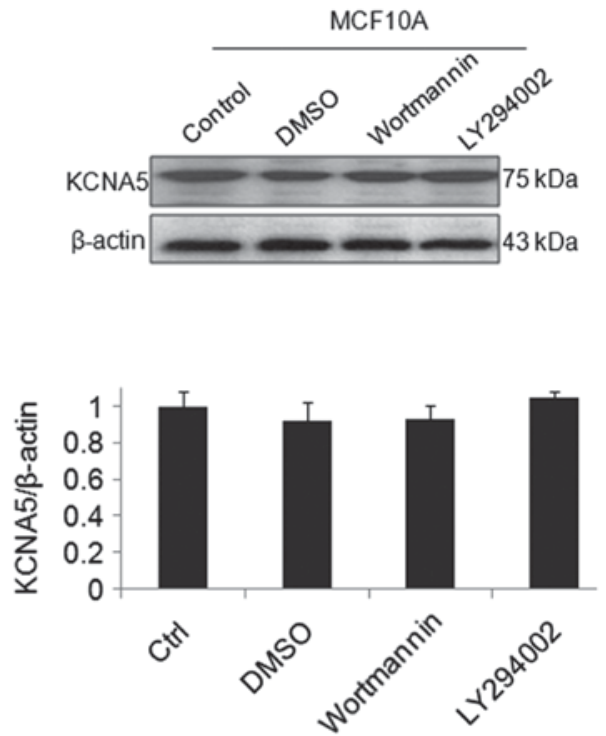

D
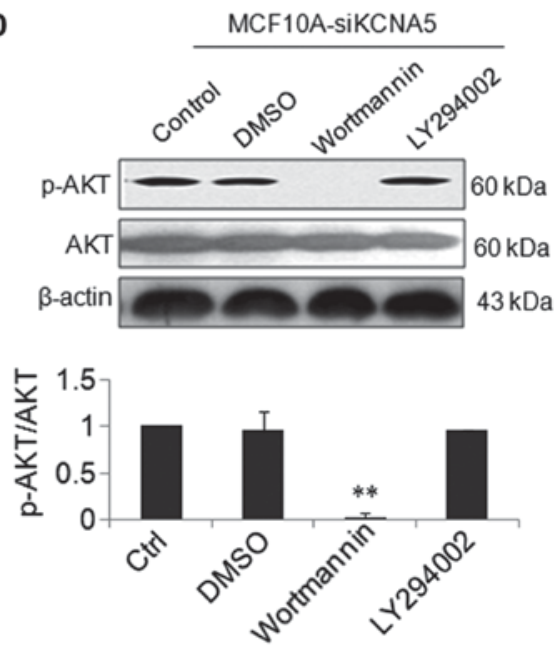

Figure 5. The PI3K/AKT signaling pathway is involved in KCNA5-dependent cell survival. Cells were cultured in serum-free medium for $4 \mathrm{~h}$, and then treated with serum for $10 \mathrm{~min}$, proteins were harvested and the expression of p-AKT and p-MAPK analyzed by western blot analysis. Statistical analyses of p-AKT and p-MAPK levels. (A) Western blot analyses of AKT, MAPK phosphorylation and PCNA expression in MCF-10A-neoT-vector (transfected with the empty expression vector) and MCF-10A-neoT-siKCNA5 (transfected with KCNA5 siRNA plamid). (B) Western blot analyses of KCNA5 expression after MCF-10A-neoT cells were treated with wortmannin $(1 \mu \mathrm{M})$ and LY294002 $(50 \mu \mathrm{M})$ for $24 \mathrm{~h}$. (C and D) Cells were cultured in serum-free medium for $4 \mathrm{~h}$, and then treated with wortmannin $(1 \mu \mathrm{M})$ and LY294002 $(50 \mu \mathrm{M})$ for $10 \mathrm{~min}$. Western blot analyses of AKT activation in MCF-10A-neoT and MCF-10A-neoT-siKCNA5. The experiment was repeated three times. ${ }^{*} \mathrm{P}<0.05$ and ${ }^{* *} \mathrm{P}<0.01$ vs. vector or Ctrl. KCNA5, potassium voltage-gated channel subfamily A member 5.

MDA-MB-231 cells. Overall, these data suggested that the expression and function of KCNA5 are related to Cav-1, and that KCNA5 facilitates the activation of AKT signaling with lipid rafts.

$\mathrm{Kv}$ channels are often observed in the MCF-7 human breast cancer cells. Abdul et al, found that the potassium channel activator, minoxidil promoted the survival of MCF-7 cells (38). Moreover, the use of specific and non-specific $\mathrm{K}^{+}$ channels blocker on MCF-7 cells resulted in apoptosis, which implicated the involvement of ATP sensitive channels, SK channels, and $\mathrm{Kv}$ channels were related to cells survival in the MCF-7 cells (39). KCNA5 was involved in the survival of many mammalian cells. Wonderlin and Strobl found that
KCNA5 was involved in survival of human breast cancer cells (40). From our previous study, Kv channels increased the survival of MCF-10A-neoT non-tumorigenic epithelial cell line (7), but the relevant mechanism is still unclear. In our present study, MCF-10A-neoT normal cells expressed high levels of KCNA5 and Cav-1 while MCF-7 human breast cancer cells expressed low levels of KCNA5 and Cav-1. We established a KCNA5 knockdown cell line with MCF-10A-neoT cells. Interestingly, we observed that KCNA5 knockdown inhibited AKT phosphorylation. And wortmannin and LY294002 PI3-kinase inhibitors have differential effects on p-AKT in MCF-10A-neoT-siKCNA5 cells. According to some studies, LY294002 potently block $\mathrm{K}_{\mathrm{v}}$ currents and promote 


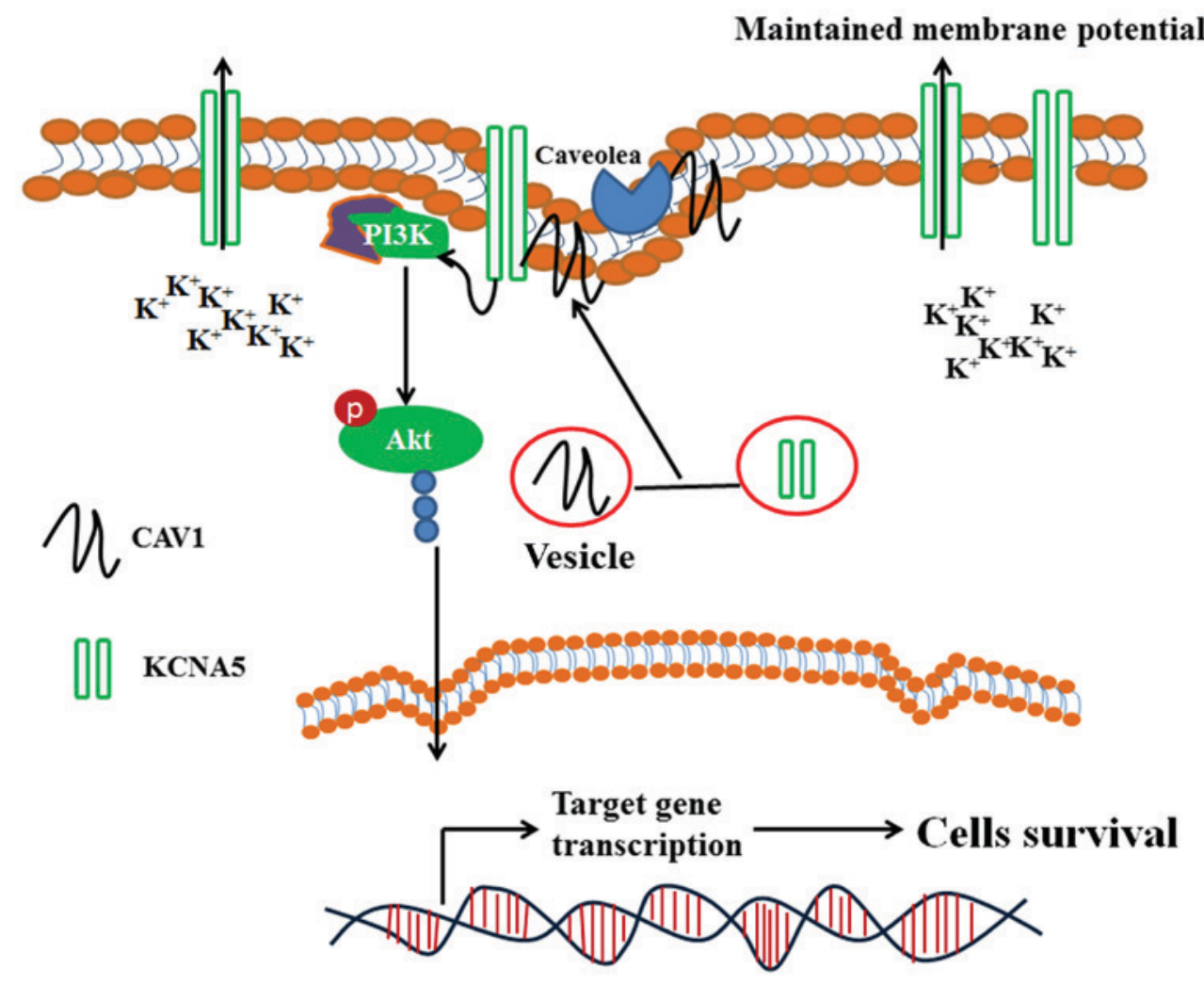

Figure 6. The proposed machanism underlying the observed cell survival effect of KCNA5 and Cav-1. Cav-1 expressed in membrane and cytoplasm and facilitated KCNA5 coupling caveoae. KCNA5 expressed in caveolae or non-caveolae. KCNA5 located in non-caveolae potentially. KCNA5 located in caveolae with Cav-1 together, which activated AKT signaling and promoted cell survival. KCNA5, potassium voltage-gated channel subfamily A member 5; Cav-1, caveolin-1.

increase in $\left[\mathrm{Ca}^{2+}\right] \mathrm{i}$ operates independently of PI3K $(35,41)$. Our result showed that LY294002 failed to activate AKT in MCF-10A-neoT-siKCNA5 cells, which is related to cell membrane depolarization and $\mathrm{Ca}^{2+}$ decrease. In MCF-7 cells, KCNA5 had no distinct effect on cell survival, but the overexpression of Cav-1 and KCNA5 promoted cell survival. It has been reported that the suppression of Cav-1 increased AKT phosphorylation facilitated the survival of MCF-10A-neoT ${ }^{\mathrm{CE}}$ cells, which downregulated Cav-1 expression, indicating that Cav-1 may be involved in KCNA5-mediated AKT phosphorylation (42). Therefore, KCNA5 is related to survival of human breast cancer cells via Cav-1 that has not been reported so far.

Cav-1, a signature protein in the integrated membrane component of caveolae, plays a scaffolding role in molecular signaling and endocytic trafficking (41). Various membrane proteins are endocytosed through a caveolin-dependent pathway, such as fibronectin, estrogen receptors, and ion channels proteins $(43,44)$. Previous evidence suggested that lipid rafts regulate channels function in different ways $(45,46)$. The interplay of ion channels and proteins, which regulates channel function, can impact the channel behavior. One important finding of our study is that Cav-1 associates with KCNA5 in order to promote cell survival, which is consistent with previous studies that Cav-1 regulates the trafficking of KCNA5 to lipid raft microdomains in cardiac cells stably transfected with human vectors of KCNA5 and caveolin (47). In our study, we found Cav-1 is mainly expressed in the cytoplasm, but there is low level of Cav-1 expressed in the cell membrane in MCF-7 cells And immunofluorescence suggested that a mass of Cav-1 and KCNA5 cannot co-express in membrane. Therefore, a large amount of KCNA5 cannot be located in caveolae. At the same time, it was showed that Cav-1 and KCNA5 were co-localized in cell membrane of MCF-10A-neoT cells, and the immunoprecipitation was also used to determine the interaction between KCNA5 and Cav-1 in MCF-10A-neoT cells (34). The MCF7 cells with KCNA5 overexpression had no effect on cell survival; but cells with both KCNA5 and Cav-1 overexpression had increased cell survival. Our study speculated that KCNA5 regulated cell survival when located in the caveolae. Moreover, cholesterol-depleting experiment showed the decreased expression of KCNA5 and Cav-1 in the plasma membrane, which indicated that channel function, is related to the cholesterol in the membrane microenvironment. Our results were consistent with ones obtained from previous studies in different cell lines $(48,49)$. Some studies have focused on the multiple function of Cav-1 in different cells and tissues, different stages of development, different physiological and pathological processes. Since 2000, our lab has been forces on the multiple function of Cav-1 in different cells and tissues, different stages of development, different physiological and pathological processes (50). To date interestingly, as functional marker, it plays a role either as a tumor suppressor or an oncogene depending on the tumor type and context of tumor progression. Disruption of Caveolae integrity or downregulation of Cav-1 appears to be a common theme in oncogenic transformation in breast cancer cells.

Disruption of Caveolae integrity or downregulation of Cav-1 appears to be a common theme in oncogenic transformation of breast cancer cells. In addition, it has been shown that 
Cav-1 can inhibit the activity of several caveolae-associated signaling molecules, including Src, H-Ras and G-proteins. On the other hand, evidence in support of an oncogenic role for Cav-1 was provided by studies showing that Cav-1 promotes cell survival in metastatic prostate cancer cells. Indeed, Cav-1 expression is upregulated in numerous cancer cell lines and tumor specimens. Our lab also reported that knockdown Cav-1 increased cell proliferation, and colony formation in mammary epithelial cells, which expression of KCNA5 is more than that in MDA-MB-231 than MCF-7 cells $(34,51,52)$. In this study, it has been shown that expression of Cav-1 was more in MDA-MB-231 metastatic breast cancer cell than MCF-7. In this study, the important interaction between KCNA5 and Cav-1 in mammary cells was showed, and it was proved that Cav-1 is required for expression of KCNA5 channel for cell survival. In addition, our data showed a strong co-expression of Cav-1 and KCNA5 in MCF-10A-neoT non-tumorigenic epithelial cell line. And it is reported that the interaction also exists in both heart and vascular smooth muscle $(28,53)$. Our results strongly suggest that the KCNA5 channel increased survival of human breast cancer cells through the PI3K/AKT signaling pathway rather than the MAPK pathway.

\section{Acknowledgements}

The authors would like to thank Dr Jie Zheng (Department of Physiology and Membrane Biology, School of Medicine, UC Davis) for providing the KCNA5 vector.

\section{Funding}

This study was supported by grants (grant nos. 30570225 and 30970353) from the National natural science foundation of China. And Science and technology plan projects in Liaoning Province, China (grant nos. L2015020568 and L201783647).

\section{Availability of data and materials}

The datasets used and/or analyzed during the current study are available from the corresponding author on reasonable request.

\section{Authors' contributions}

$\mathrm{CQ}$, JS, and YL were involved in conceptualization of the study, data acquisition, formal analysis, and writing of the original draft. XW, CH, LW and QC were involved in methodology planning and data analysis. TG, YZ, HL and YW analyzed data. WZ and JL designed the project, and acquired funding and resources.

\section{Ethics approval and consent to participate}

Patients' relatives provided written informed consent for the procedures. The present study was approved by the Ethics Committee of The First Affiliated Hospital of Dalian Medical University for the use of human subjects.

\section{Consent for publication}

Not applicable.

\section{Competing interests}

The authors declare that they have no competing interests.

\section{References}

1. Wulff H, Castle NA and Pardo LA: Voltage-gated potassium channels as therapeutic targets. Nat Rev Drug Discov 8: 982-1001, 2009.

2. Kunzelmann K: Ion channels and cancer. J Membr Biol 205: 159-173, 2005.

3. Lee JH, Park JW, Byun JK, Kim HK, Ryu PD, Lee SY and Kim DY: Silencing of voltage-gated potassium channel KV9.3 inhibits proliferation in human colon and lung carcinoma cells. Oncotarget 6: 8132-8143, 2015.

4. Delgado-Ramírez M, Morán-Zendejas R, Aréchiga-Figueroa IA Toro-Castillo C, Ramírez-Martínez JF and RodríguezMenchaca AA: Modulation of the voltage-gated potassium channel Kv2.1 by the anti-tumor alkylphospholipid perifosine. Pharmacol Rep 68: 457-461, 2016.

5. Tegla CA, Cudrici C, Rozycka M, Soloviova K, Ito T, Singh AK, Khan A, Azimzadeh P, Andrian-Albescu M, Khan A, et al: C5b-9-activated, K(v)1.3 channels mediate oligodendrocyte cell cycle activation and dedifferentiation. Exp Mol Pathol 91: 335-345, 2011.

6. Kazama I, Baba A, Matsubara M, Endo Y, Toyama H and Ejima Y: Benidipine suppresses in situ proliferation of leukocytes and slows the progression of renal fibrosis in rat kidneys with advanced chronic renal failure. Nephron Exp Nephrol 128: 67-79, 2014.

7. Liu J, Feng S, Zhang L, Wu Z, Chen Q, Cheng W, Wang SQ and Zou W: Expression and properties of potassium channels in human mammary epithelial cell line MCF10A and its possible role in proliferation. Sheng Li Xue Bao 62: 203-209, 2010 (In Chinese).

8. Ru Q, Tian X, Wu YX, Wu RH, Pi MS and Li CY: Voltage-gated and ATP-sensitive $\mathrm{K}+$ channels are associated with cell proliferation and tumorigenesis of human glioma. Oncol Rep 31: 842-848, 2014.

9. Pincus DW, DiCicco-Bloom E and Black IB: Role of voltage-sensitive calcium channels in mitogenic stimulation of neuroblasts. Brain Res 553: 211-214, 1991.

10. Wei T, Liang Z, Jin Y and Zhang L: Effect of berberine, liensinine and neferine on HERG channel expression. Zhongguo Zhong Yao Za Zhi 38: 239-244, 2013 (In Chinese).

11. Zhu YX, Yin H, Bruins LA, Shi CX, Jedlowski P, Aziz M, Sereduk C, Kortuem KM, Schmidt JE, Champion M, et al: RNA interference screening identifies lenalidomide sensitizers in multiple myeloma, including RSK2. Blood 125: 483-491, 2015.

12. Guo TB, Lu J, Li T, Lu Z, Xu G, Xu M, Lu L and Dai W: Insulin-activated, $\mathrm{K}^{+}$-channel-sensitive Akt pathway is primary mediator of ML-1 cell proliferation. Am J Physiol Cell Physiol 289: C257-C263, 2005.

13. Roderick C, Reinach PS, Wang L and Lu L: Modulation of rabbit corneal epithelial cell proliferation by growth factor-regulated $\mathrm{K}(+)$ channel activity. J Membr Biol 196: 41-50, 2003.

14. Ballou LM, Lin RZ and Cohen IS: Control of cardiac repolarization by phosphoinositide 3-kinase signaling to ion channels. Circ Res 116: 127-137, 2015.

15. Shepherd AJ, Loo L and Mohapatra DP: Chemokine co-receptor CCR5/CXCR4-dependent modulation of Kv2.1 channel confers acute neuroprotection to HIV-1 glycoprotein gp120 exposure. PLoS One 8: e76698, 2013.

16. Harder T and Simons K: Caveolae, DIGs, and the dynamics of sphingolipid-cholesterol microdomains. Curr Opin Cell Biol 9: 534-542, 1997.

17. Takaguri A, Kamato M, Satoh Y, Ohtsuki K and Satoh K: Effect of alteration of caveolin-1 expression on doxorubicin-induced apoptosis in H9c2 cardiac cells. Cell Biol Int 39: 1053-1060, 2015.

18. Banadakoppa M, Goluszko P, Liebenthal D and Yallampalli C: Nitric oxide induces segregation of decay accelerating factor (DAF or CD55) from the membrane lipid-rafts and its internalization in human endometrial cells. Cell Biol Int 36: 901-907, 2012.

19. Takaguri A, Kamato M, Satoh Y, Ohtsuki K and Satoh K: Effect of alteration of caveolin-1 expression on doxorubicin-induced apoptosis in H9c2 cardiac cells. Cell Biol Int 39: 1053-1060, 2015.

20. Cheng ZJ, Singh RD, Marks DL and Pagano RE: Membrane microdomains, caveolae, and caveolar endocytosis of sphingolipids. Mol Membr Biol 23: 101-110, 2006. 
21. Parton RG and Simons K: The multiple faces of caveolae. Nat Rev Mol Cell Biol 8: 185-194, 2007.

22. Lapierre LA, Ducharme NA, Drake KR, Goldenring JR and Kenworthy AK: Coordinated regulation of caveolin-1 and Rab1la in apical recycling compartments of polarized epithelial cells. Exp Cell Res 318: 103-113, 2012.

23. Shvets E, Ludwig A and Nichols BJ: News from the caves: Update on the structure and function of caveolae. Curr Opin Cell Biol 29: 99-106, 2014.

24. Thomas CM and Smart EJ: Caveolae structure and function. J Cell Mol Med 12: 796-809, 2008.

25. Thompson MA, Prakash YS and Pabelick CM: The role of caveolae in the pathophysiology of lung diseases. Expert Rev Respir Med 8: 111-122, 2014.

26. Chaudhary KR, Cho WJ, Yang F, Samokhvalov V, El-Sikhry HE, Daniel EE and Seubert JM: Effect of ischemia reperfusion injury and epoxyeicosatrienoic acids on caveolin expression in mouse myocardium. J Cardiovasc Pharmacol 61: 258-263, 2013.

27. Martens JR, Sakamoto N, Sullivan SA, Grobaski TD and Tamkun MM: Isoform-specific localization of voltage-gated $\mathrm{K}^{+}$ channels to distinct lipid raft populations. Targeting of Kv1.5 to caveolae. J Biol Chem 276: 8409-8414, 2001.

28. Cogolludo A, Moreno L, Lodi F, Frazziano G, Cobeño L, Tamargo J and Perez-Vizcaino F: Serotonin inhibits voltage-gated $\mathrm{K}^{+}$currents in pulmonary artery smooth muscle cells: Role of 5-HT2A receptors, caveolin-1, and KV1.5 channel internalization. Circ Res 98: 931-938, 2006.

29. Brignell JL, Perry MD, Nelson CP, Willets JM, Challiss RA and Davies NW: Steady-state modulation of voltage-gated $\mathrm{K}^{+}$ channels in rat arterial smooth muscle by cyclic AMP-dependent protein kinase and protein phosphatase 2B. PLoS One 10 e0121285, 2015.

30. Brazer SC, Singh BB, Liu X, Swaim W and Ambudkar IS: Caveolin-1 contributes to assembly of store-operated $\mathrm{Ca}^{2+}$ influx channels by regulating plasma membrane localization of TRPC1. J Biol Chem 278: 27208-27215, 2003

31. Anderson RG: The caveolae membrane system. Annu Rev Biochem 67: 199-225, 1998.

32. Okamoto T, Schlegel A, Scherer PE and Lisanti MP: Caveolins, a family of scaffolding proteins for organizing 'preassembled signaling complexes' at the plasma membrane. J Biol Chem 273: 5419-5422, 1998 .

33. Overmiller AM, McGuinn KP, Roberts BJ, Cooper F, Brennan-Crispi DM, Deguchi T, Peltonen S, Wahl JK II and Mahoney MG: c-Src/Cav-1-dependent activation of the EGFR by Dsg2. Oncotarget 7: 37536-37555, 2016.

34. Feng S, Wang Y, Wang X, Wang Z, Cui Y, Liu J, Zhao C, Jin M and Zou W: Caveolin-1 gene silencing promotes the activation of PI3K/AKT dependent on Eralpha36 and the transformation of MCF10ACE. Sci China Life Sci 53: 598-605, 2010.

35. Wang X, Feng S, Zhang H, Wang Y, Cui Y, Wang Z, Liu J and Zou W: RNA inference-mediated caveolin-1 downregulation decrease estrogen receptor alpha $(E R \alpha)$ signaling in human mammary epithelial cells. Mol Biol Rep 38: 761-768, 2011.

36. Liu J, Qu C, Li H, Zhang Y, Sun J, Yang S, Liu J, An L and Zou W: Expression of KCNA5 protein in human mammary epithelial cell line associated with caveolin-1. J Membr Biol 249: 449-457, 2016.

37. Qu C, Fu XZ, Han C, Chen Q, Liu Y, Wang Xb, Xi RG, Liu J and Zou W: Voltage-gated potassium channel KV1.5 protects against $\mathrm{MPP}^{+}$mediated neurotoxicity in PC12 cells. Adv Biochem 2: 103-108, 2014.

38. Abdul M, Santo A and Hoosein N: Activity of potassium channel-blockers in breast cancer. Anticancer Res 23: 3347-3351, 2003 .
39. Zou W, Zhang L, Wang X and Zhou SS: Effect of potassium channel antagonist on proliferation in the human mammary epithelial cells MCF10A. J Liao Ning Norm Univ 31: 23-26, 2008.

40. Wonderlin WF and Strobl JS: Potassium channels, proliferation and G1 progression. J Membr Biol 154: 91-107, 1996.

41. El-Kholy W, Macdonald PE, Lin JH, Wang J, Fox JM, Light PE, Wang Q, Tsushima RG and Wheeler MB: The phosphatidylinositol 3-kinase inhibitor LY294002 potently blocks K(V) currents via a direct mechanism. FASEB J 17: 720-722, 2003.

42. Nauc V, De Lamirande E, Leclerc P and Gagnon C: Inhibitors of phosphoinositide 3-kinase, LY294002 and wortmannin, affect sperm capacitation and associated phosphorylation of proteins differently: $\mathrm{Ca}^{2+}$-dependent divergences. J Androl 25: 573-585, 2004.

43. Cipriani P, Di Benedetto P, Capece D, Zazzeroni F, Liakouli V, Ruscitti P, Pantano I, Berardicurti O, Carubbi F, Alesse E and Giacomelli R: Impaired Cav-1 expression in SSc mesenchymal cells upregulates VEGF signaling: A link between vascular involvement and fibrosis. Fibrogenesis Tissue Repair 7: 13, 2014.

44. Sottile J and Chandler J: Fibronectin matrix turnover occurs through a caveolin-1-dependent process. Mol Biol Cell 16: 757-768, 2005.

45. Martens JR, Navarro-Polanco R, Coppock EA, Nishiyama A Parshley L, Grobaski TD and Tamkun MM: Differential targeting of Shaker-like potassium channels to lipid rafts. J Biol Chem 275: 7443-7446, 2000.

46. Markandeya YS, Phelan LJ, Woon MT, Keefe AM, Reynolds CR, August BK, Hacker TA, Roth DM, Patel HH and Balijepalli RC: Caveolin-3 overexpression attenuates cardiac hypertrophy via inhibition of T-type $\mathrm{Ca}^{2+}$ current modulated by protein kinase ca in cardiomyocytes. J Biol Chem 290: 22085-22100, 2015.

47. Schilling JM, Horikawa YT, Zemljic-Harpf AE, Vincent KP, Tyan L, Yu JK, McCulloch AD, Balijepalli RC, Patel HH and Roth DM: Electrophysiology and metabolism of caveolin-3-overexpressing mice. Basic Res Cardiol 111: 28, 2016.

48. Vicente R, Villalonga N, Calvo M, Escalada A, Solsona C, Soler C, Tamkun MM and Felipe A: Kv1.5 association modifies Kv1.3 traffic and membrane localization. J Biol Chem 283: 8756-8764, 2008.

49. Pérez-Verdaguer M, Capera J, Martínez-Mármol R, Camps M, Comes N, Tamkun MM and Felipe A: Caveolin interaction governs Kv1.3 lipid raft targeting. Sci Rep 6: 22453, 2016.

50. Zou W, Mcdaneld L and Smith LM: Caveolin 1 haploinsufficiency leads to partial transformation of human breast epithelial cells. Anticancer Res 23: 4581-4586, 2003.

51. Méndez-Bolaina E, Sánchez-González J, Ramírez-Sánchez I, Ocharán-Hernández E, Núñez-Sánchez M, Meaney-Mendiolea E, Meaney A, Asbun-Bojalil J, Miliar-García A, Olivares-Corichi I and Ceballos-Reyes G: Effect of caveolin-1 scaffolding peptide and 17 beta-estradiol on intracellular $\mathrm{Ca}^{2+}$ kinetics evoked by angiotensin II in human vascular smooth muscle cells. Am J Physiol Cell Physiol 293: C1953-C1961, 2007.

52. Ishikawa T, Yuhanna IS, Umetani J, Lee WR, Korach KS, Shaul PW and Umetani M: LXR $\beta /$ estrogen receptor- $\alpha$ signaling in lipid rafts preserves endothelial integrity. J Clin Invest 123: 3488-3497, 2013.

53. Abi-Char J, Maguy A, Coulombe A, Balse E, Ratajczak P, Samuel JL, Nattel S and Hatem SN: Membrane cholesterol modulates Kv1.5 potassium channel distribution and function in rat cardiomyocytes. J Physiol 582: 1205-1217, 2007.

This work is licensed under a Creative Commons Attribution-NonCommercial-NoDerivatives 4.0 International (CC BY-NC-ND 4.0) License. 